\title{
The place of CEUS in distinguishing benign from malignant cervical lymph nodes: a prospective study
}

\author{
Laura Poanta ${ }^{1}$, Oana Serban ${ }^{1}$, Isabela Pascu ${ }^{1}$, Sever Pop ${ }^{2}$, Marcel Cosgarea ${ }^{2}$, Daniela Fodor ${ }^{1}$
}

${ }^{1} 2^{\text {nd }}$ Internal Medicine Department, ${ }^{2}$ ENT Department, "Iuliu Hatieganu” University of Medicine and Pharmacy, Cluj-Napoca, Romania

\begin{abstract}
Aims: The aim of the study was to evaluate the ability of contrast-enhanced ultrasonography (CEUS) compared to grayscale B-mode and color Doppler ultrasound in differentiate benign versus malign superficial cervical lymph nodes. Material and methods: In a prospective study ultrasonography (gray scale, color and spectral Dopller, and CEUS) was performed in 61 patients (33 men, 28 women; mean age of 51.2 years, range: 18-81 years), with cervical lymphadenopathy. The nodes were examined and biopsied or surgically removed. CEUS was performed with $2.4 \mathrm{ml}$ intravenous bolus of contrast agent Sono Vue and the results were registered with a special software. Results: Of all the nodes, 32 were benign and 29 were malignant (metastases). Solbiati index was higher in benign nodes $(2.23 \pm 0.84$ vs $1.50 \pm 0.48, \mathrm{p}<0.05)$. Doppler parameters (vessel location, vascular pattern, pedicullum number, resistivity index, and pulsatility index) were significantly lower in benign nodes $(\mathrm{p}<0.001)$, and ROC analysis returned excellent results. For CEUS, derived peak intensity (DPI \%) was higher in benign nodes $(17.72 \pm 5.43$ vs $11.76 \pm 4.88, \mathrm{p}<0.05)$; regional blood volume $(\mathrm{RBV})$ was also higher $(849.8 \pm 467.1 \mathrm{vs} 458.3 \pm 283.3$, $\mathrm{p}<0.05$ ). The time to peak (TTP, s) and area under the curve (AUC, cm2) were similar in both benign and malignant nodes. Enhancement pattern was the most accurate to characterize benign versus malignant nodes. Sensitivity and specificity were higher for DPI, RBV and enhancement pattern from CEUS, according to ROC analysis, compared to gray scale ultrasound, but lower than color Doppler. Analyzing the place of CEUS in lymph node evaluation we found that CEUS is most useful for the evaluation of the lymph nodes with uncertain aspect at gray scale and Doppler evaluation. Conclusions: ROC analysis confirmed the higher degree of diagnostic accuracy of CEUS in comparison with conventional techniques for some parameters such as enhancement pattern. Evaluation of nodal perfusion with this method can be helpful in the differentiation of benign from malignant nodes but requires further confirmation.
\end{abstract}

Keywords: lymph nodes, ultrasonography, gray scale, Doppler, CEUS

\section{Introduction}

Ultrasound (US) is the commonly used method for evaluating superficial lymph nodes and it is also important in staging nodal metastases [1,2]. It is widely available, easy to use, and less expensive than other imaging

Received 16.12.2013 Accepted 18.01.2014

Med Ultrason

2014, Vol. 16, No 1, 7-14

Corresponding author: Daniela Fodor, MD, PhD

$2^{\text {nd }}$ Internal Medicine Department

"Iuliu Hatieganu" University of Medicine and

Pharmacy, Cluj-Napoca, Romania

2-4 Clinicilor str

400006 Cluj Napoca, Romania

Phone: 004 0264591942/442

Email: dfodor@umfcluj.ro methods, and it has the benefit of nonionizing radiation. Accurate lymph node characterization is important for a wide number of clinical situations, including prognosis prediction, selecting and monitoring treatment, beyond the diagnosis itself (cancer, lymphoma, or inflammatory nodes) $[1,3]$. The differences between benign and malignant nodes are reported on the basis of various diagnostic criteria in gray scale US, such as the shape, border, echogenicity, calcification, necrosis, or vascularization pattern by Doppler US examination. Malignant nodes are described to be hypoechoic, without echogenic hilum, with round shape, longitudinal to transverse diameters ratio less than 2, and blood vessels predominant in periphery [3-7]. Contrast-enhanced ultrasound (CEUS) is a modern imaging method evaluating tissue perfusion in real time. The administration of contrast agent intrave- 
nously and special soft of ultrasound device is necessary $[8,9]$. In our prospective study we analyzed the usefulness of CEUS in distinguishing between benign and malignant lymph nodes, evaluating the nodes perfusion and enhancement pattern compared with conventional US, in the differential diagnosis of the superficial lymphadenopathy. The results were compared with the histological examination of the nodes.

\section{Material and methods}

Prospectively, 61 patients ( 33 men, 28 women; mean age of 51.2 years, range: $18-81$ years) were examined by US (gray scale, Doppler, and CEUS) between September 2012-March 2013 in the $2^{\text {nd }}$ Internal Department. The majority of the patients were referred for US examination from ENT department due to the existence of palpable mass/masses in the cervical region. For this reason all the malignant nodes represented metastasis from ENT cancers. In cases with multiple lymphadenomegaly the most representative lymph node was chosen for analysis. The diagnoses were confirmed by US guided biopsy or by surgical removal of the lymph node and subsequent histological examination. Pediatric patients and patients with lymphoma were excluded from the study. The local Ethics Committee approved the study, and all participants gave their written informed consent prior to the investigation.

\section{Gray scale and color Doppler US examination}

All the patients were examined with a Logiq S8 GE ultrasound machine with a high-frequency linear probe $(6-15 \mathrm{MHz})$.

In gray scale the following parameters were recorded: the longitudinal and transverse diameters (for Solbiati index calculation - the ratio between the longitudinal and the transversal axis), the echogenicity of the nodes (classified as hypoechoic, isoechoic, and hyperechoic), hilum visibility (present, hard to see, or absent), margins (sharp, irregular, blurred), and homogeneity or internal structure changes (calcification, necrosis).

In Doppler US (pulse repetition frequency $350 \mathrm{~Hz}$, wall filter $45 \mathrm{~Hz}$ ) three patterns of nodal vascularization were defined: 1) hilar pattern with flow signals in the nodal hilum, 2) peripheral pattern with flow signals mainly in peripheral nodal parts, and 3) mixed pattern with both hilar and peripheral pattern. The resistivity index (RI) and pulsatility index (PI) were measured in the main artery of the node.

\section{CEUS examination}

For CEUS examination $(3-9 \mathrm{MHz}$ linear probe, range of gain: $80-94 \%$, compression 36 , mechanical index 0.07 ) a bolus of $2.4 \mathrm{ml}$ of contrast agent (Sono-

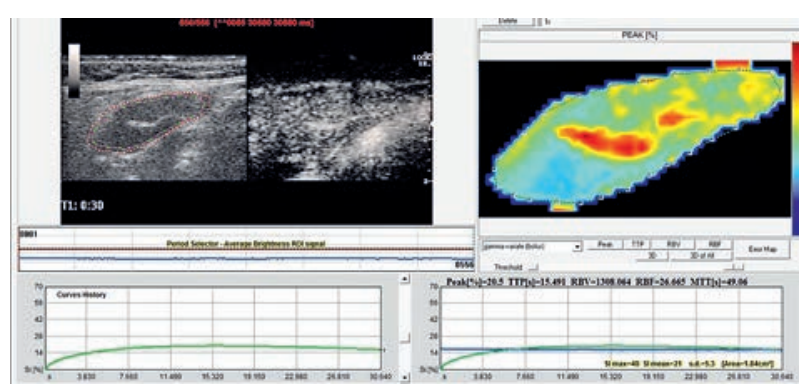

Fig 1. CEUS examination, peak of signal intensity map, and CEUS parameters of an inflammatory lymph node.

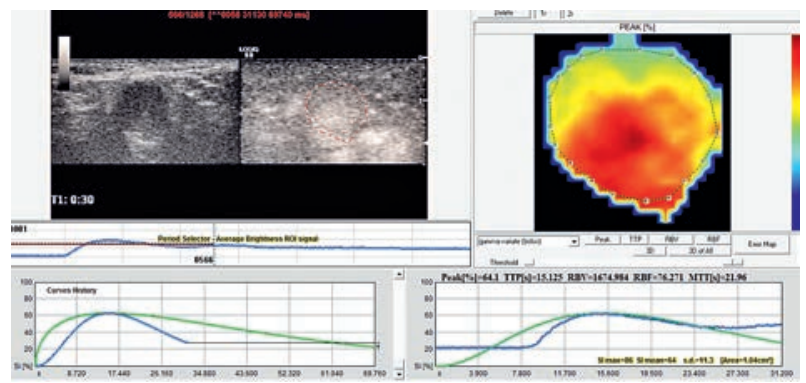

Fig 2. CEUS examination, peak of signal intensity map, and CEUS parameters of a metastasis from laryngeal cancer.

Vue, Bracco Imaging SpA, Milan, Italy) was administered intravenously, followed by the flushing of $10 \mathrm{ml}$ saline solution. All CEUS examinations were digitally recorded. The nodal perfusion and the enhancement pattern were evaluated in the arterial (10-15 s after bolus of contrast agent) and parenchymal (15-30 s after bolus of contrast agent) phase. Four nodal enhancement patterns were defined: 1) intense homogeneous enhancement, 2) moderate homogeneous enhancement, 3) inhomogeneous enhancement with perfusion defects, and 4) lack of enhancement. Using Qontrast software (Esaote S.p.a, Florence, Italy) for perfusion quantification the derived peak intensity (DPI - \%), time to peak (TTP- seconds), regional blood volume (RBV) and area under the curve $\left(\right.$ AUC $-\mathrm{cm}^{2}$ ) were measured (fig 1, fig 2).

After the histological examination of the biopsy material or of the surgical removed node, the examined cervical lymph nodes were divided into two groups: benign and malignant nodes and the US findings were compared with histological diagnosis.

\section{Statistical analysis}

Standard descriptive statistics were adopted for the analysis; we defined means and standard deviations for numeric results. T-test was used to compare means. Chisquare was used to compare the categorical parameters. ROC (Receiver Operating Characteristic) analysis was 
Table I. Lymph nodes characterization by gray scale ultrasound.

\begin{tabular}{|c|c|c|c|}
\hline $\begin{array}{l}\text { Ultrasound } \\
\text { characteristic n(\%) }\end{array}$ & $\begin{array}{l}\text { Benign nodes } \\
(\mathrm{N}=32)\end{array}$ & $\begin{array}{l}\text { Malignant nodes } \\
(\mathrm{N}=29)\end{array}$ & P value \\
\hline $\mathrm{SI}(\mathrm{L} / \mathrm{T} \geq 2)$ & $18(56.2)$ & $2(6.8)$ & $<0.001$ \\
\hline \multicolumn{4}{|l|}{ Echogenicity } \\
\hline Hypoechic & $8(25)$ & $18(62)$ & \multirow{3}{*}{0.011} \\
\hline Isoechoic & $17(53.1)$ & $9(31)$ & \\
\hline Hyperechoic & 7 (21.8) & $2(6.8)$ & \\
\hline \multicolumn{4}{|l|}{ Hilum visibility } \\
\hline Present & $24(75)$ & $11(37.9)$ & \multirow{3}{*}{$<0.001$} \\
\hline Hard to see & $8(25)$ & $15(51.7)$ & \\
\hline Absent & 0 & $3(10.3)$ & \\
\hline \multicolumn{4}{|l|}{ Internal structure } \\
\hline Homogenous & $25(78.1)$ & $6(20.6)$ & \multirow{4}{*}{$<0.001$} \\
\hline Inhomogeneous & $7(21.8)$ & $21(72.4)$ & \\
\hline Necrosis & 0 & 0 & \\
\hline Calcifications & 0 & $2(6.8)$ & \\
\hline \multicolumn{4}{|l|}{ Margins } \\
\hline Sharp & $26(81.2)$ & $15(51.7)$ & \multirow{3}{*}{0.009} \\
\hline Irregular & 0 & $5(17.3)$ & \\
\hline Blurred & $6(18.8)$ & $9(31)$ & \\
\hline
\end{tabular}

Legend: $\mathrm{SI}=$ Solbiati index. $\mathrm{L} / \mathrm{T}=$ ratio between longitudinal and transverse diameters

used for the evaluation of sensitivity, specificity, positive and negative predictive value and overall ability of contrast-enhanced ultrasonography, gray-scale B-mode and power Doppler parameters to distinguish between benign and malignant nodes by calculating the area under the curve (we named it AUROC not to be confused with CEUS software measured parameter AUC). $\mathrm{P}$ value was considered significant at a value lower than 0.05 . The results among all the investigation techniques were compared in order to establish a possible examination protocol and the place of CEUS in lymph nodes US investigation. Microsoft Office Excel 2003 and SPSS for Windows version 19 was used.

\section{Results}

\section{Gray scale examination}

The gray scale characteristics of the lymph nodes are detailed in table I. Mean L/T ratio was higher in the benign nodes ( $\mathrm{p}<0.001)$. Most of the benign nodes were isoor hyperechoic (total 24 nodes, over $70 \%$ ), while $62 \%$ of the malignant nodes were hypoechoic. Hilum was visible in $75 \%$ of the benign nodes, but in only $37.9 \%$ of the malignant nodes. Most of the benign nodes were homog- enous, without necrosis or calcifications, and with sharp margins. Most of the malignant nodes were inhomogeneous, a minority had calcifications, and almost half of them had irregular or blurred margins.

\section{Color Doppler examination}

Color Doppler US showed different patterns in malignant versus benign nodes, (table II). Also, the mean values for RI and PI were lower in benign lymph nodes: $0.53 \pm 0.06$ in benign nodes vs $0.69 \pm 0.07$ in malignant nodes ( $\mathrm{p}<0.001)$, and $0.82 \pm 0.16$ in benign nodes vs 1.37 \pm 0.35 in malignant nodes, respectively $(p<0.001)$. All of the benign nodes had hilar and regular vascular pattern, with only one pedicullum ( 31 of 32 nodes). More than half of the malignant nodes had peripheral or mixed vessels with chaotic patterns, and multiple pedicullus.

\section{CEUS examination}

CEUS enhancement patterns and perfusion quantification are shown in table III. DPI and RBV are significantly higher in benign nodes, while AUC and TTP showed similar results. The majority of the benign nodes showed homogenous enhancement patterns, while the malignant nodes showed mostly inhomogeneous patterns and even one node with no enhancement. 
ROC analysis was performed for numerical values (table IV, fig 3 and 4 ).

To find the utility of the three US methods for correct identification of benign $v s$ malignant nodes, the results were combined. Parameters used for analysis were the parameters found to have statistically significance in comparison benign $v s$ malignant: for gray scale- $\mathrm{L} / \mathrm{T}$ ratio, echogenicity, homogeneity, margins and hilum; for Doppler- vessel location, vascular pattern, pedicullum number, RI (cutoff value of 0.605 obtained from ROC analysis) and PI (cutoff value of 0.995 obtained from ROC analysis); and for CEUS- enhancement pattern, PI (cutoff value of 14.15 obtained from ROC analysis) and RBV (cutoff value of 497 obtained from ROC analysis).
If the gray scale US was combined with Doppler, all benign lymph nodes were correctly identified $(100 \%)$, but only 22 from 29 malignant lymph nodes (75.8\%). Six out of 29 malignant nodes were uncertain (20.7\%) and one lymph node was incorrectly identified as benign (3.5\%). When CEUS was added, five out of the six uncertain lymph nodes were correctly identified. It means that if all the three methods were combined $100 \%$ of benign nodes were correctly identified and 93.1\% (27 from 29) of malignant nodes.

Considering the aforementioned parameters we developed a score that could be used to distinguish between malignant and benign lymph nodes using gray scale, Doppler and CEUS (table V).

Table II. Lymph nodes characterization by color Doppler - vascular pattern.

\begin{tabular}{|c|c|c|c|c|c|c|c|}
\hline Nodes & $\begin{array}{l}\text { Hilar } \\
\text { pattern }\end{array}$ & $\begin{array}{l}\text { Peripheral } \\
\text { pattern }\end{array}$ & $\begin{array}{l}\text { Mixed } \\
\text { pattern }\end{array}$ & $\begin{array}{l}\text { Regular } \\
\text { pattern }\end{array}$ & $\begin{array}{l}\text { Chaotic } \\
\text { pattern }\end{array}$ & $\begin{array}{l}\text { One } \\
\text { pedicullum }\end{array}$ & $\begin{array}{l}\text { Multiple } \\
\text { pedicullus }\end{array}$ \\
\hline Benign $(\mathrm{N}=32)$ & $32(100)$ & 0 & 0 & $32(100)$ & 0 & $31(96.8)$ & $1(3.1)$ \\
\hline Malignant $(\mathrm{N}=29)$ & $13(44.8)$ & $7(24.1)$ & $9(31)$ & $9(31)$ & $20(68.9)$ & $12(41.4)$ & $17(58.6)$ \\
\hline P value & & $<0.001$ & & \multicolumn{2}{|c|}{$<0.001$} & \multicolumn{2}{|c|}{$<0.001$} \\
\hline
\end{tabular}

The results are expressed in number $(\%)$

Table III. Lymph nodes characterization by CEUS - enhancement homogeneity and the visibility of hilar artery.

\begin{tabular}{llll}
\hline $\begin{array}{l}\text { CEUS } \\
\text { characteristic }\end{array}$ & $\begin{array}{l}\text { Benign nodes } \\
(\mathbf{N}=\mathbf{3 2})\end{array}$ & $\begin{array}{l}\text { Malignant nodes } \\
(\mathbf{N = 2 9 )}\end{array}$ & p value \\
\hline Intense homogeneous & $20(62.5)$ & $4(13.8)$ & \\
Mild homogeneous & $9(28.1)$ & $5(17.2)$ & $<\mathbf{0 . 0 0 1}$ \\
Inhomogeneous & $3(9.3)$ & $19(65.5)$ & \\
No enhancement & 0 & $1(3.4)$ & \\
DPI (\%) & $17.72 \pm 5.43$ & $11.76 \pm 4.88$ & $<\mathbf{0 . 0 0 1}$ \\
TTP (sec) & $16.16 \pm 2.90$ & $17.12 \pm 2.45$ & $\mathbf{0 . 1 8 9}$ \\
AUC (cm2) & $1.11 \pm 0.61$ & $1.83 \pm 1.73$ & $\mathbf{0 . 0 3 6}$ \\
RBV & $849.8 \pm 467.1$ & $458.3 \pm 283.3$ & $<\mathbf{0 . 0 5}$ \\
\hline
\end{tabular}

The results are expressed in number $(\%)$ or in number \pm standard deviation. Legend: DPI $=$ derived peak intensity, TTP $=$ time to peak, $\mathrm{AUC}=$ area under the curve, $\mathrm{RBV}=$ regional blood volume

Table IV. ROC analysis: benign versus malignant nodes.

\begin{tabular}{lccccccc}
\hline Parameters & AUROC value & P value & Statistical Sign** & CI 95\% & Cutoff value & Sensitivity (\%) & Specificity (\%) \\
\hline L/T ratio* & 0.745 & 0.001 & Fair & $0.612-0.878$ & $2 *$ & 93 & 53 \\
RI & 0.945 & 0.000 & Excellent & $0.890-0.999$ & 0.605 & 90.3 & 84.4 \\
PI & 0.927 & 0.000 & Excellent & $0.865-0.990$ & 0.995 & 87.1 & 84.2 \\
RBV & 0.746 & 0.001 & Fair & $0.620-0.872$ & 497 & 75.9 & 75 \\
DPI (\%) & 0.805 & 0.000 & Good & $0.696-0.914$ & 14.15 & 75.9 & 75 \\
\hline
\end{tabular}

Legend: $*$ for $\mathrm{L} / \mathrm{T}$ the cutoff value is pre-established, $* *$ according to the following classification: $0.500-0.600=$ fail, $0.600-0.700=$ poor, $0.700-0.800=$ fair, $0.800-0.900=$ good, $0.900-1=$ excellent. $\mathrm{L} / \mathrm{T}$ ratio $=$ ratio between longitudinal and transverse diameters, $\mathrm{RI}=$ resistivity index, $\mathrm{PI}=$ pulsatility index, $\mathrm{RBV}=$ regional blood volume, $\mathrm{DPI}=$ derived peak intensity . 


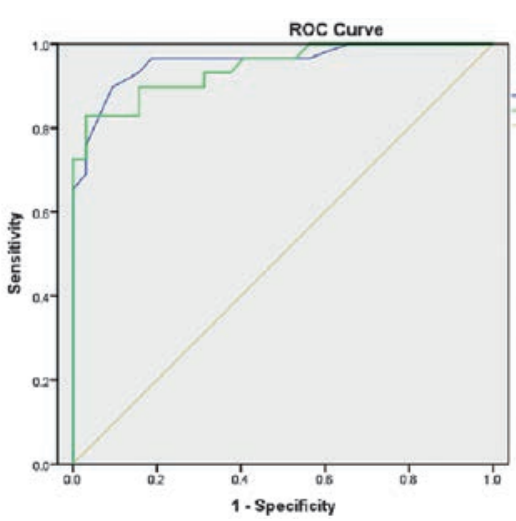

Fig 3. ROC analysis for color Doppler (RI, resistivity index and PI, pulsatility index)

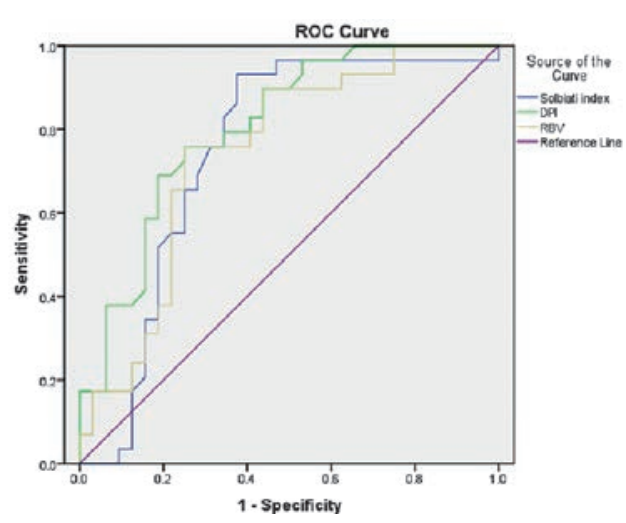

Fig 4. ROC analysis for gray scale and CEUS (DPI, derived pulsatility index, RBV, regional blood volume)

Table V. The score for distinguishing between malignant and benign nodes using gray scale US, color Doppler US, and CEUS.

\begin{tabular}{|c|c|c|}
\hline Criteria & Characteristics & Number of Points \\
\hline \multicolumn{3}{|l|}{ Gray scale US } \\
\hline \multirow[t]{2}{*}{ 1. $\mathrm{L} / \mathrm{T}$ ratio } & $\geq 2$ & 0 \\
\hline & $<2$ & 1 \\
\hline \multirow[t]{2}{*}{ 2. Echogenicity } & Isoechoic/Hyperechoic & 0 \\
\hline & Hypoechoic & 1 \\
\hline \multirow[t]{2}{*}{ 3. Internal structure } & Homogenous & 0 \\
\hline & Inhomogeneous/Necrosis/ Calcifications & 1 \\
\hline \multirow[t]{2}{*}{ 4. Margins } & Sharp & 0 \\
\hline & Irregular/Blurred & 1 \\
\hline \multirow[t]{2}{*}{ 5. Hilum } & Present & 0 \\
\hline & Hard to see/Absent & 1 \\
\hline \multicolumn{3}{|l|}{ Color Doppler US } \\
\hline \multirow[t]{2}{*}{ 6. Vessel location } & Hilar pattern & 0 \\
\hline & Peripheral pattern/Mixed pattern & 1 \\
\hline \multirow[t]{2}{*}{ 7. Vascular pattern } & Regular pattern & 0 \\
\hline & Chaotic pattern & 1 \\
\hline \multirow[t]{2}{*}{ 8. Pedicullum number } & One & 0 \\
\hline & Multiple & 1 \\
\hline \multirow[t]{2}{*}{ 9. RI } & $<0.605$ & 0 \\
\hline & $\geq 0.605$ & 1 \\
\hline \multirow[t]{2}{*}{ 10. PI } & $<0.995$ & 0 \\
\hline & $\geq 0.995$ & 1 \\
\hline Score $<5$ - benign node & Score $=5-$ CEUS needed & it node \\
\hline \multicolumn{3}{|l|}{ CEUS } \\
\hline \multirow[t]{2}{*}{ Enhancement pattern } & Homogenous & 0 \\
\hline & Inhomogeneous/ No enhancement & 1 \\
\hline \multirow[t]{2}{*}{ DPI } & $\geq 14.15$ & 0 \\
\hline & $<14.15$ & 1 \\
\hline \multirow[t]{2}{*}{ RBV } & $\geq 497$ & 0 \\
\hline & $<497$ & 1 \\
\hline
\end{tabular}

Legend: $\mathrm{RI}=$ resistivity index, $\mathrm{PI}=$ pulsatility index $\mathrm{DPI}=$ derived peak intensity, $\mathrm{RBV}=$ regional blood volume 
If, after gray scale US and Doppler examination, less than 5 criteria are accomplished ( $<5$ points), the nodes should be interpreted as benign. If more than 5 criteria are met ( $>5$ points), the node should be interpreted as malignant. When the node accomplishes 5 criteria (5 points), it should be interpreted as uncertain and CEUS should be performed. After CEUS, if the node meets at least 2 criteria ( $\geq 2$ points) it should be considered malignant.

\section{Discussions}

The role of US in the evaluation of superficial lymph nodes is well known and established [4,5]. It allows a detailed evaluation of nodes, including nodal internal structure and it seems to be superior to computed tomography (CT) for superficial lymph nodes [10]. The main utility of $\mathrm{CT}$ is the detection of deeply located nodes, especially in cervical region [10]. However, the limits of US are often discussed, such as incapacity to detect blood flow in all intranodal blood vessels by color and power Doppler $[11,12]$.

Yu et al [13] studied 94 enlarged superficial lymph nodes. Regarding gray scale ultrasound, they showed that an L/T ratio of 2 or less has low specificity and accuracy. They found that $\mathrm{L} / \mathrm{T}$ ratio is one of the less valuable parameters in the evaluation of lymph nodes. In our study, at a cut off value of 2, we have a very high sensitivity $(93 \%)$ but a very low specificity (53\%). Cutoff level of $\mathrm{L} / \mathrm{T}$ ratio is 2 in most of the studies using gray scale US and it is already accepted that nodes with $\mathrm{LT}$ ratio $\geq 2$ are classified as benign, while nodes with LT ratio $<2$ are malignant [14]. As for echogenicity, hypoechoic nodes are generally considered to be malignant. Also, absent hilum is suggestive for malignancy $[7,14]$. But it is important to note that in half of the malignant nodes in our study hilum was hard to see, so the results may be biased.

AUROC for L/T ratio, echogenicity, and hilum visibility returned values between 0.700 and 0.800 , which, according to statistical significance, indicate a fair accuracy in distinguishing between the two groups of lymph nodes. So, gray B mode US is fairly reliable in describing lymph nodes, but has some limitations; it seems that grey scale US can identify malignant nodes but cannot correctly identify the lymph nodes as non-malignant. Other studies showed that CEUS improves the specificity in diagnosing benign lymph nodes as compared to B-mode US. It does not improve the correct identification of malignant lymph nodes and cannot replace EUS-guided fine-needle aspiration [15].

In our study, all the benign nodes showed hilar vascularity with a regular pattern. RI and PI were significantly higher in malignant nodes and ROC analysis showed excellent results, which mean that this method could be considered as an excellent technique for differentiating benign from malignant nodes.

Currently used contrast agents allow a better description of microvascular pattern and can detect avascular areas of necrosis and tumoral metastasis [16]. In malignant nodes, neo-vessels with pathological aberrant feeding vessels are present, the growth being provided by angiogenetic factors.

On the basis of the presence or absence of tumor angiogenesis there are various enhancement patterns of benign and malignant nodes described in literature [16-18]: benign nodes as intense homogeneous with no perfusion defects and with visible hilum artery, probably due to rich cortical capillary vascularity [19] or inhomogeneous enhancement and perfusion defects, with no visible artery in hilum, for malignant nodes [16-18].

Rubaltelli et al. [20] investigated the lymph nodes with focal cortical thickening. They found perfusion defects of thickened cortex in nodes affected by metastasis of cutaneous melanoma. They documented high specificity of CEUS, proved by histopathology. In our study the sensitivity and specificity were not as high as the Rubatelli et al study, but we have similar results for enhancement patterns. We found the majority of benign lymph nodes to have homogenous enhancement on CEUS, either intense or moderate ( 29 of 32 nodes) and only three showed inhomogeneous enhancement. According to many authors $[8,9,20]$ normal and reactive lymph nodes have intense vascularization with cortical capillary circulation and this fact could explain the homogenous aspect. On the other hand 19 (65.5\%) of malignant nodes in our study, were inhomogeneous, and one of them had no enhancement, probably due to the fact that they were less vascularized and had perfusion defects [20]. CEUS is clinically valuable for microvascular analysis as it can deliver better data about vascular pattern and vascular defects. But few reports about the use of CEUS in superficial lymphadenopathy have been published and if they were, with controversial results [13].

ROC analysis for DPI and RBV returned good and fair results, respectively, but the values were significantly higher in benign nodes. According to some studies, TTP and AUC are considered to be lower in malignant nodes, but for DPI and RBV literature data are still contradictory $[1,20,21]$.

Other studies [22] showed no differences between CEUS parameters (DPI, TTP and AUC) in benign versus malignant nodes, but only for the difference between maximum and minimum signal intensity (SImax - SImin). According to Quifang et al [22] CEUS patterns 
differ considerably in benign versus malignant as nodes with metastases are vascularized with a heterogeneous centripetal enhancement pattern, and benign nodes have a homogenous centrifugal enhancement pattern. They underlined that even in metastatic nodes blood flow may be decreased due to vascular compression by neoplastic tissue, or vascular encasement. The blood in metastatic nodes may be affected also by encasement of the surrounding parenchyma [22]. This may explain the fact that peak intensity (DPI) varies widely among studies.

In our study, DPI and RBV were significantly lower in malignant nodes, while TTP and AUC were higher, but the difference between the two groups was statistically significant only for AUC. According to ROC analysis, these last two parameters are not useful in distinguishing between benign and malignant nodes, but AUROC had a good value for DPI and a fair value for RBV. The fact that TTP and AUC were higher in our study may be related to the technique we chose (the selection of the whole lymph node instead of a limited region of interest - ROI).

We established the criteria we used for malignancy from existing literature $[8,9,23,24]$ and we also established cutoff values for RI, PI, DPI and RBV, from our ROC analysis. When we compared the results from all three techniques used in our study, we demonstrated that the correct diagnosis of uncertain nodes after grey scale and Doppler US was significantly increased by adding CEUS. This conclusion may be biased by the small number of patients. The main conclusion drawn from this study is that CEUS should be not performed as a routine examination, but only in these cases where the results are uncertain after using both gray scale and Doppler US.

The technique used in CEUS evaluation is very important, and the results may be influenced by the contrast agent used, patient characteristics and metabolism, and the selection of ROI. A limitation of this study, beside the small number of patients, is the lack of comparison with lymphoma lymph nodes.

\section{Conclusions}

In our study we identified different enhancement patterns in benign and malignant cervical lymph nodes. Our results showed a higher degree of diagnostic accuracy of CEUS in comparison with gray scale US, although the results obtained from ROC curves are not excellent. We did not demonstrate the superiority of CEUS over color Doppler for the assessment of nodal perfusion but CEUS can be helpful in the evaluation of nodal architecture and in the detection of abnormal vascular patterns, especially where the results are not certain. We found that Doppler US (color and spectral) is the best method for differen- tiation in the benign versus malignant nodes, but more studies are necessary to establish the best ROI, the cutoff values for CEUS parameters, and to describe more accurately the enhancement patterns.

\section{Conflict of interest: none}

\section{References}

1. Slaisova R, Benda K, Jarkovsky J, Petrasova H, Szturz $\mathrm{P}$, Valek V. Contrast-enhanced ultrasonography compared to gray-scale and power doppler in the diagnosis of peripheral lymphadenopathy. Eur J Radiol 2013; 82: 693-698.

2. Vassallo P, Wernecke K, Roos N, Peters PE. Differentiation of benign from malignant superficial lymphadenopathy:the role of high-resolution US. Radiology 1992; 183: 215-220.

3. Van den Brekel MW, Stel HV, Castelijns JA, et al. Cervical lymph node metastasis: assessment of radiologic criteria. Radiology 1990; 177: 379-384.

4. Ying M, Ahuja A. Sonography of neck lymph nodes. Part I: normal lymph nodes. Clin Radiol 2003; 58: 351-358.

5. Ahuja A, Ying M. Sonography of neck lymph nodes. Part II: abnormal lymph nodes. Clin Radiol 2003; 58: 359-366.

6. Solbiati L, Cioffi V, Ballarati E. Ultrasonography of the neck. Radiol Clin North Am 1992; 30: 941-954.

7. Dudea SM, Lenghel M, Botar-Jid C, Vasilescu D, Duma M. Ultrasonography of superficial lymph nodes: benign vs. malignant. Med Ultrason 2012; 4; 294-306.

8. Ahuja A, Ying M, HO SY, et al. Ultrasound of malignant cervical lymph nodes. Cancer Imaging 2008; 8: 48-56.

9. Cui XW, Jenssen C, Saftoiu A, Ignee A, Dietrich CF. New ultrasound techniques for lymph node evaluation. World J Gastroenterol 2013; 19: 4850-4860.

10. Rubaltelli L, Proto E, Salmaso R, Bortoletto P, Candiani F, Cagol P. Sonography of abnormal lymph nodes in vitro: correlation of sonographic and histologic findings. AJR Am J Roentgenol 1990; 155: 1241-1244.

11. Tschammler A, Ott G, Schang T, Seelbach-Goebel B, Schwager K, Hahn D. Lymphadenopathy: differentiation of benign from malignant disease-color Doppler US assessment of intranodal angioarchitecture. Radiology 1998; 208: 117-123.

12. Ariji Y, Kimura Y, Hayashi N, et al. Power Doppler sonography of cervical lymph nodes in patients with head and neck cancer. AJNR Am J Neuroradiol 1998; 19: 303-307.

13. Yu M, Liu Q, Song HP, et al. Clinical application of contrast-enhanced ultrasonography in diagnosis of superficial lymphadenopathy. J Ultrasound Med 2010; 29: 735-740.

14. Wunderbaldinger P. Problems and prospects of modern lymph node imaging. Eur J Radiol 2006; 58: 325-337.

15. Hocke M, Menges M, Topalidis T, Dietrich CF, Stallmach A. Contrast-enhanced endoscopic ultrasound in discrimination between benign and malignant mediastinal and abdominal lymph nodes. J Cancer Res Clin Oncol 2008; 134 : 473-480. 
16. Solbiati L, Cova L. Improved characterization of reactive and malignant lymph nodes using contrast-enhanced ultrasound. In: Albrecht T, Thorelius L, Solbiati L, Cova L, Frauscher F. (eds). Contrast-enhanced ultrasound in clinical practice. Liver, prostate, pancreas, kidney and lymph nodes.Springer-Verlag, Milan 2005: 39-50.

17. Döme B, Hendrix MJ, Paku S, Tóvári J, Tímár J. Alternative vascularization mechanisms in cancer: Pathology and therapeutic implications. Am J Pathol 2007; 170: 1-15.

18. Rubaltelli L, Khadivi Y, Tregnaghi A, et al. Evaluation of lymph node perfusion using continuous mode harmonic ultrasonography with a second-generation contrast agent. J Ultrasound Med 2004; 23: 829-836.

19. Gadre A, Briner W, O’Leary M. A scanning electron microscope study of the human cercical lymph node. Acta Otolaryngol 1994; 114: 87-90.

20. Rubaltelli L, Beltrame V, Tregnaghi A, Scagliori E, Frigo AC, Stramare R. Contrast-enhanced ultrasound for char- acterizing lymph nodes with focal cortical thickening in patients with cutaneous melanoma. AJR Am J Roentgenol 2011; 196: W8-W12.

21. Wilson SR, Greenbaum LD, Goldberg BB. Contrast-enhanced ultrasound: what is the evidence and what are the obstacles? AJR Aam J roentgenol 2009; 193: 55-60.

22. Ouyang Q, Chen L, Zhao H, Xu R, Lin Q. Detecting metastasis of lymph nodes and predicting aggressiveness in patients with breast carcinomas. J Ultrasound Med 2010; 29: 343-352.

23. Na GD, Lim HK, Byun HS, Kim HD, Ko YH, Baek JH. Differential diagnosis of cervical lymphadenopathy: usefulness of color Doppler sonography. AJR Am J Roentgenol 1997; 168: 1311-1316.

24. Ouyang Q, Chen L, Zhao H, Xu R, Lin Q. Detecting metastasis of lymph nodes and predicting aggressiveness in patients with breast carcinomas. J Ultrasound Med 2010; 29: 343-352. 